\title{
Higher-order approximation techniques for estimating stochastic parameter of a sediment transport model
}

\author{
F.-C. Wu, C.-K. Wang
}

Abstract: Higher-order approximation techniques for estimating stochastic parameter of the non-homogeneous Poisson (NHP) model are presented. The NHP model is characterized by a two-parameter cumulative probability distribution function (CDF) of sediment displacement. Those two parameters are the temporal and spatial intensity functions, physically representing the inverse of the average rest period and step length of sediment particles, respectively.

Difficulty of estimating the parameters has, however, restricted the applications of the NHP model. The approximation techniques are proposed to address such problem. The basic idea of the method is to approximate a model involving stochastic parameters by Taylor series expansion. The expansion preserves certain higher-order terms of interest. Using the experimental (laboratory or field) data, one can determine the model parameters through a system of equations that are simplified by the approximation technique. The parameters so determined are used to predict the cumulative distribution of sediment displacement. The second-order approximation leads to a significant reduction of the CDF error (of the order of 47\%) compared to the first-order approximation. Error analysis is performed to evaluate the accuracy of the first- and second-order approximations with respect to the experimental data. The higher-order approximations provide better estimations of the sediment transport and deposition that are critical factors for such environment as spawning gravel-bed.

Key words: Non-homogeneous Poisson model, parameter estimation, approximation technique, Taylor series, cumulative probability distribution function, intensity function, error analysis.

Fu-Chun Wu

Department of Agricultural Engineering and Hydrotech Research Institute, National Taiwan University, Taipei, Taiwan, 10617, Republic of China

Chi-Kuei Wang

Department of Agricultural Engineering,

National Taiwan University, Taipei, Taiwan, 10617,

Republic of China

Correspondence to: Fu-Chun Wu, Fax ++886 22369 2466, e-mail: fcwu@hy.ntu.edu.tw

The work presented herein is supported by the National Science Council of the Republic of China (Grant No. NSC-86-2611-E-002036T). Gratitude is due to Mr. Sen-Hae Yeh for his assistance on the flume experiments. The invaluable comments and suggestions of the anonymous reviewers are sincerely appreciated. 


\section{Introduction}

Stochastic processes have been used for modeling sediment transport over six decades. Einstein (1937) presented perhaps, the first stochastic model for sediment transport. His model was based on the concept that bed-load particles moving in a sequence of alternate steps and rests. Since then, a number of stochastic models have been proposed. For example, the two-layer model by Crickmore and Lean (1962), the exponential-exponential model by Hubbell and Sayre (1964), the gamma-exponential model by Yang and Sayre (1971), and the model of Vukmirovic and Wilson (1977) which stated the influence of the present step on the probability of next step [see Hung and Shen (1972); Shen and Cheong (1980) for further discussions]. Among them, the result presented by Hubbell and Sayre (1964) is considered to coincide with Einstein's model. Both used the simplified flow conditions and assumed a homogeneous process of transport.

Shen and Todorovic (1971) eliminated certain idealized assumptions to develop a general stochastic model for one-dimensional movement of bed material. They regarded the transport of sediment as a non-homogeneous random process and hypothesized the rest periods and step lengths of sediment particles being temporal and spatial varying quantities, respectively. The non-homogeneous Poisson (NHP) model of Shen-Todorovic is described by the cumulative probability distribution function (CDF) of sediment displacement:

$$
\begin{aligned}
F_{t}(x)= & \operatorname{Prob}\left(X_{t} \leq x\right) \\
= & \exp \left[-\int_{t_{0}}^{t} \lambda_{1}(\tau) \mathrm{d} \tau\right] \cdot \exp \left[-\int_{x_{0}}^{x} \lambda_{2}(\xi) \mathrm{d} \xi\right] \\
& \cdot \sum_{n=0}^{\infty} \sum_{j=n}^{\infty} \frac{\left[\int_{t_{0}}^{t} \lambda_{1}(\tau) \mathrm{d} \tau\right]^{n}}{n !} \frac{\left[\int_{x_{0}}^{x} \lambda_{2}(\xi) \mathrm{d} \xi\right]^{j}}{j !} \\
= & \exp \left[-\Lambda_{1}(t)\right] \cdot \exp \left[-\Lambda_{2}(x)\right] \cdot \sum_{n=0}^{\infty} \sum_{j=n}^{\infty} \frac{\left[\Lambda_{1}(t)\right]^{n}}{n !} \frac{\left[\Lambda_{2}(x)\right]^{j}}{j !}
\end{aligned}
$$

in which $X_{t}$ denotes the x-direction displacement of a particle at time $t ; \lambda_{1}$ and $\lambda_{2}$ are the temporal and spatial intensity functions, physically representing the inverse of the average rest period and step length; $\Lambda_{1}$ and $\Lambda_{2}$ are the corresponding integral intensity functions. The homogeneous model of Einstein-Hubbell-Sayre may be viewed as a special case of this more general yet less restrictive stochastic model. Mathematically the NHP model is an improvement over the earlier ones. However, the complexity of this model and difficulty in determining the parameters restricts its broad applications.

A first-order approximation technique for estimating the parameters of the NHP model is proposed to address the above problem (Wu and Shen, 1998). The basic idea of such method is to approximate a model involving stochastic parameters by the first-order Taylor expansions. Although the proposed approximation technique shows significant progress and the results are encouraging, the $\mathrm{CDF}$ error of the first-order approximation (sometimes exceeds $10 \%$ with respect to the measured data) is not negligible for certain critical situations. For instance, the survival of salmon eggs deposited in the gravel is of important ecological and economic concerns, the NHP model may well be used to simulate the sediment transport and resulting deposition in the spawning gravels. The egg survival is 
related to the apparent velocity through the spawning gravels (Cooper, 1965), a $10 \%$ deviation in the specific deposit of sediment leads to a $40 \%$ difference in the survival rate (Milhous, 1982). Such inaccuracy leads to a marked overestimate on either the cost required for habitat conservation or the production of fishes. Since the error term associated with the first-order Taylor expansions is of the second order of a finite increment (i.e. $O\left(\Delta t^{2}\right)$ or $O\left(\Delta x^{2}\right)$ ), one can improve the accuracy of the approximation method by either reducing the size of the increment or preserving the higher-order terms in Taylor series. The latter is adopted in the present study because it prevents increasing the difficulties of experimental measurement and makes more significant improvement than the former.

This work presents the second- and higher-order approximation techniques for estimating the stochastic parameter of the NHP model. Flume studies are carried out to verify the proposed approximation scheme. The results of the first- and secondorder approximations are compared. The general form of the simplified CDF for higher-order approximations is provided. Error analysis is performed to evaluate the accuracy of the first- and second-order approximations.

\section{2}

\section{Higher-order approximation techniques}

The first-order approximation technique indeed provides a route to approach the temporal and spatial intensity functions. However, it is noticed that $\Lambda_{1}$ is the dominating term that governs the temporal variation of Eq. (1). In addition, the successive measurements of sediment distribution at a small time interval, $\Delta t$, are practically inefficient and physically infeasible without an adequate experimental apparatus. The first writer and Shen (1998) recommended a simple alternative method to evaluate the integral temporal intensity function. Therefore, the main focus of this paper is the estimation of the spatial intensity function using higherorder approximation techniques based on Taylor's expansions.

\section{1}

\section{The second-order approximation technique}

To develop the second-order approximation technique, the CDF of sediment displacement is expanded both forwards and backwards with respect to a selected point $x$ by the finite increments, $\Delta x$ and $2 \Delta x$. This is accomplished by introducing the second-order Taylor series of the integral spatial intensity function into the forward- and backward-expansions of Eq. (1). The second-order expansions are divided by the CDF at $x$ for further simplification. The simplified forward- and backward-expansions are solved as a system to evaluate the parameter $\lambda_{2}$. Such system of equations is listed below and its derivation is shown in Appendix A.

$$
\begin{aligned}
& \frac{F_{t_{i}}(x+\Delta x)}{F_{t_{i}}(x)}=\exp \left(-a_{1}-a_{2}\right) \cdot\left[1+\left(a_{1}+a_{2}\right) \cdot b_{1}+\frac{1}{2} a_{1}^{2} \cdot b_{2}\right] \\
& \frac{F_{t_{i}}(x-\Delta x)}{F_{t_{i}}(x)}=\exp \left(a_{1}-a_{2}\right) \cdot\left[1+\left(-a_{1}+a_{2}\right) \cdot b_{1}+\frac{1}{2} a_{1}^{2} \cdot b_{2}\right] \\
& \frac{F_{t_{i}}(x+2 \Delta x)}{F_{t_{i}}(x)}=\exp \left(-2 a_{1}-4 a_{2}\right) \cdot\left[1+\left(2 a_{1}+4 a_{2}\right) \cdot b_{1}+2 a_{1}^{2} \cdot b_{2}\right] \\
& \frac{F_{t_{i}}(x-2 \Delta x)}{F_{t_{i}}(x)}=\exp \left(2 a_{1}-4 a_{2}\right) \cdot\left[1+\left(-2 a_{1}+4 a_{2}\right) \cdot b_{1}+2 a_{1}^{2} \cdot b_{2}\right]
\end{aligned}
$$


in which $a_{1}, a_{2}, b_{1}$, and $b_{2}$, all varying as a function of $x$, are the four unknowns to be solved. The quantitative values of $F_{t_{i}}(x-2 \Delta x)$ through $F_{t_{i}}(x+2 \Delta x)$ are determined from the data taken at a specific time $t_{i}$. Since that $a_{1}=\Lambda_{2}^{\prime} \cdot \Delta x$ (see Appendix A) and $\Lambda_{2}^{\prime}=\lambda_{2}$, one can determine the magnitude of $\lambda_{2}$ at the location $x$ once $a_{1}$ is solved. Eventually, one can pursue the spatial intensity function by solving the system of equations incrementally at the locations where the cumulative distribution of sediment is measured.

\section{2}

\section{Higher-order approximation techniques}

Following the procedures described in the preceding section, one can essentially develop the higher-order approximation techniques for estimating the parameters of the NHP model (Wu and Wang, 1997). Preserving the higher-order terms in Taylor expansions, however, complicates the derivation and increases the number of unknowns. Table 1 is a summary of the simplified form of the expanded CDF. For the ith-order approximation method, as indicated in Table 1, the number of unknowns (or equations) to be solved is $2 i$. These unknowns are $a_{1}$ through $a_{i}$ and $b_{1}$ through $b_{i}$. The number of locations where the cumulative distributions of sediment are to be measured is $2 i+1$, namely, $(x-i \Delta x)$ through $(x+i \Delta x)$. For example, to collect the physical data required for the second-order approximation, the sampling points must be extended both forwards and backwards by an additional increment.

\section{3}

\section{Laboratory experiments}

The transport and resulting deposition of sediment in the gravel-bed rivers is an issue attracting considerable concerns. The intrusion of fine sand into spawning gravels and the pollution of streambed by contaminated sediment are commonly reported [e.g. Lisle (1989); Jobson and Carey (1989); Joy et al. (1993)]. Modeling the spatiotemporal distribution of sediment in such environment is essential for assessing the consequential impacts. The aim of this experimental study is mainly to verify the proposed approximation techniques and grasp a better understanding of the physical process. The experiments are conducted in a tilting flume of $20 \mathrm{~cm} \times 40 \mathrm{~cm}$ cross-section. The bottom of the flume is paved with 5-cm-thick gravel substrate, as shown in Figure 1. A sand strip of the predetermined amount is placed across an upstream section of the gravel bed and subjected to a steady flow. For each trial, flow is terminated after a period of running and the water is drained. Several samples are then taken longitudinally

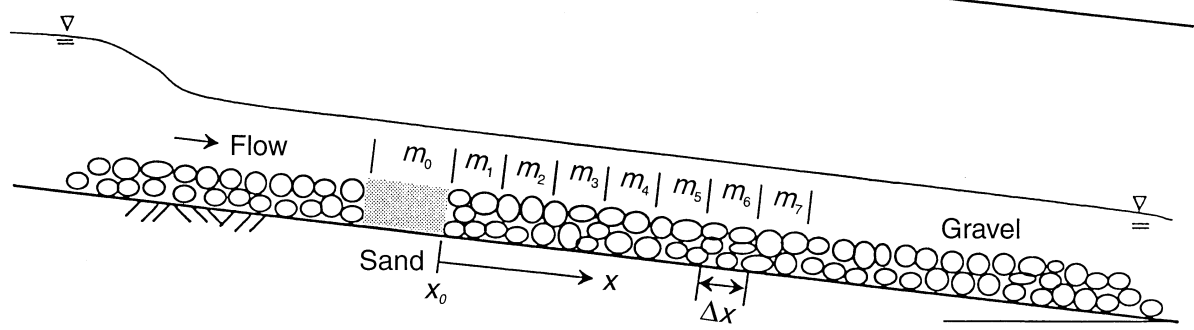

Fig. 1. Schematic diagram of experimental setup and configuration 


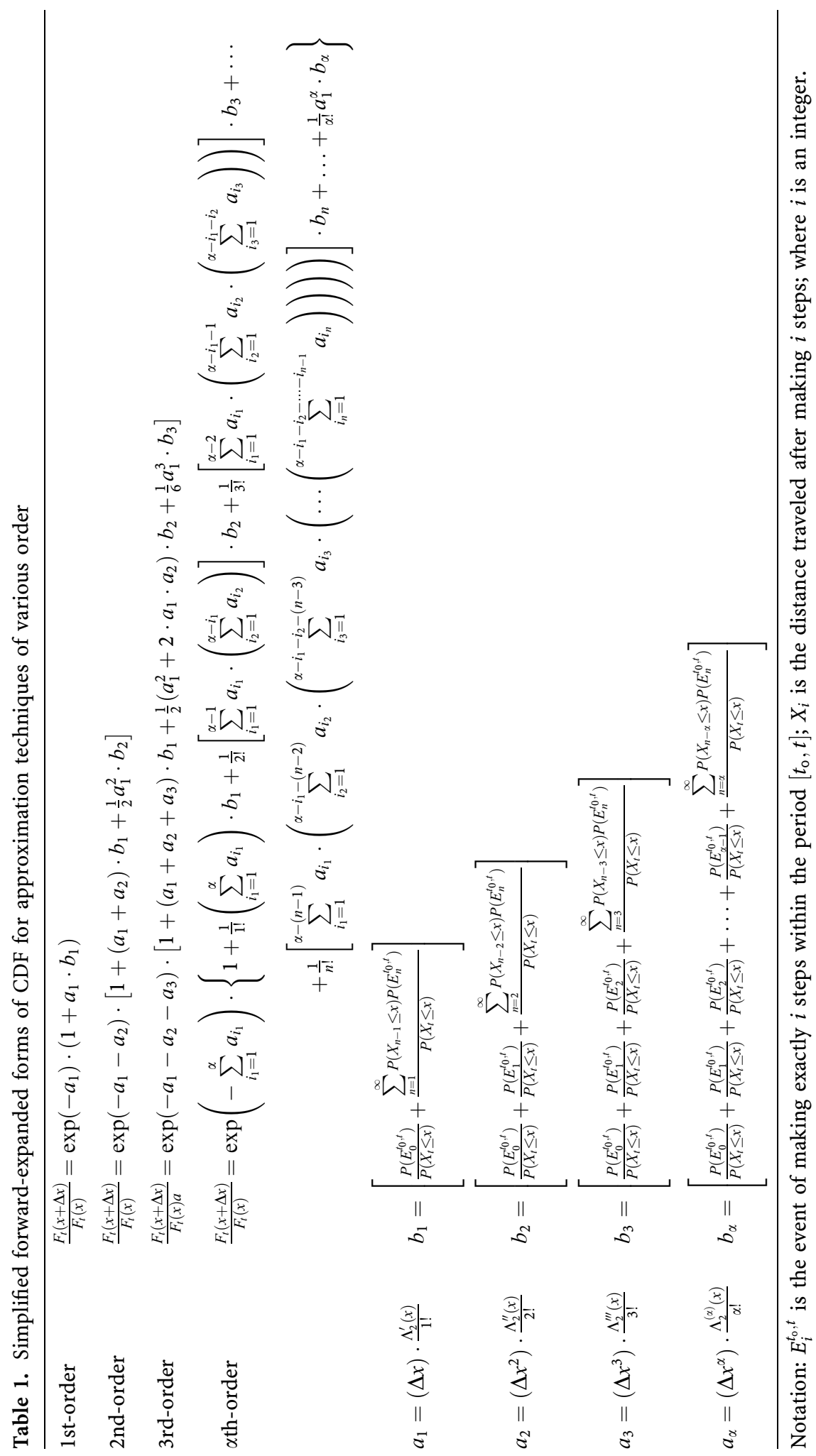


along the flume with an interval of $8 \mathrm{~cm}$ (designated as $\Delta x$ in Fig. 1). The quantities of sediment remained at the source and within the gravel substrate (designated as $m_{0}$ and $m_{1}$ through $m_{7}$, respectively) are physically measured. Two types of uniformly-graded sand with $d_{50}$ of $0.65 \mathrm{~mm}$ (type C) and $0.30 \mathrm{~mm}$ (type F) are used. The well-sorted gravel with $D_{50}$ of $8.3 \mathrm{~mm}$ is used as the substrate. Flowrate is in the range between $1 \times 10^{-3}$ and $4 \times 10^{-3} \mathrm{cms}$, slope of the flume varies from $6 / 1000$ to $20 / 1000$. A complete list of the testing conditions and the experimental results is given in Table 2 . The cumulative probability of sediment distribution can be determined precisely from the data measured for a specific period of running, i.e.

$F_{t}\left(x_{n}\right)=\frac{\sum_{i=0}^{n} m_{i}}{M_{T}}$

where $x_{n}=n \cdot \Delta x$ for $n=0,1,2, \ldots, 7 ; M_{T}(=1 \mathrm{~kg})$ is the quantity of sand introduced at the upstream source. The cumulative distribution of sediment evaluated for various locations is then substituted into Eqs. (2)-(5) to solve the model parameters.

4

\section{Results and discussion}

Based on the experimental data, one can determine the spatial intensity function and consequently calculate the cumulative probability distribution of sediment displacement. The first- and second-order approximations are presented and compared in the following sections.

\section{1}

\section{Spatial intensity function}

The magnitudes of the spatial intensity function at several locations from the source are estimated by the first- and second-order approximation techniques. Fig. 2 illustrates the calculated $\lambda_{2}$ and the fitting curves. These curves are typically of exponential distribution, i.e.

$\lambda_{2}(x)=a \cdot \mathrm{e}^{-b x}$

in which $a$ and $b$ are fitting coefficients. As $\lambda_{2}$ is varying as a function of distance only, a series of experiments for various running periods, carried out with identical testing conditions, corresponds to one spatial intensity function. Fig. 2 reveals that the exponent $b$ is nearly identical for both the first- and second-order curves, but the coefficient $a$ is smaller for the higher-order approximation. Further study is required to investigate if this is a universal law for the third- and higher-order methods. However, according to a similar experimental study ( $\mathrm{Wu}$, 1993), it is anticipated that the stochastic parameter varies with the physical properties such as the ratio of gravel to sediment sizes, the amount of sediment introduced, the slope of the flume and the flowrate. The monotonously descending trend of the $\lambda_{2}$ curve (or the increasing trend of the step length) is attributed to the increasing void space, along the flume, that is available for sediment particles to move through.

\section{2}

\section{Cumulative probability distribution of sediment displacement}

The first- and second-order approximations of the spatial intensity function are used to calculate the cumulative probability distributions of sediment displace- 


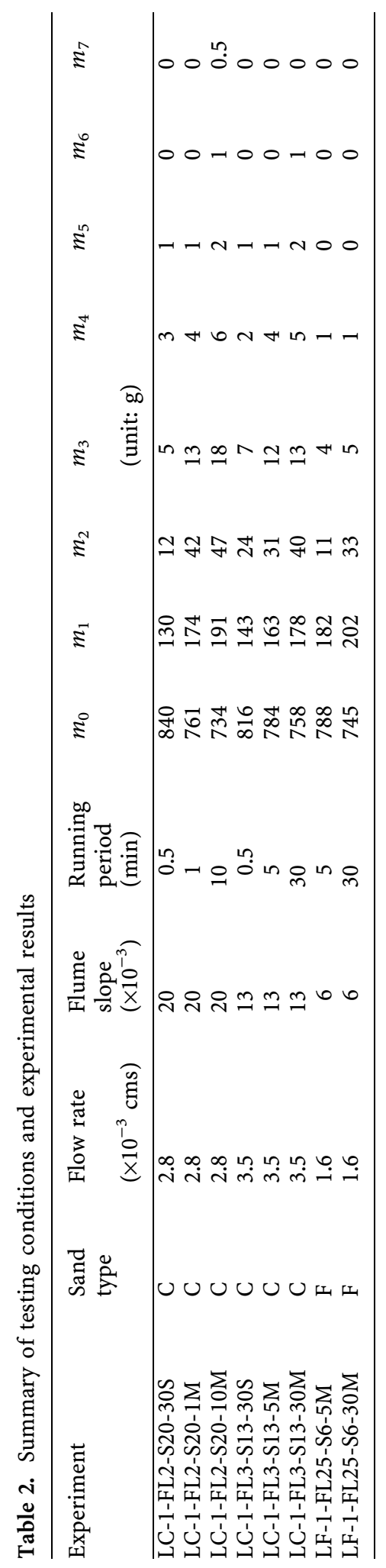



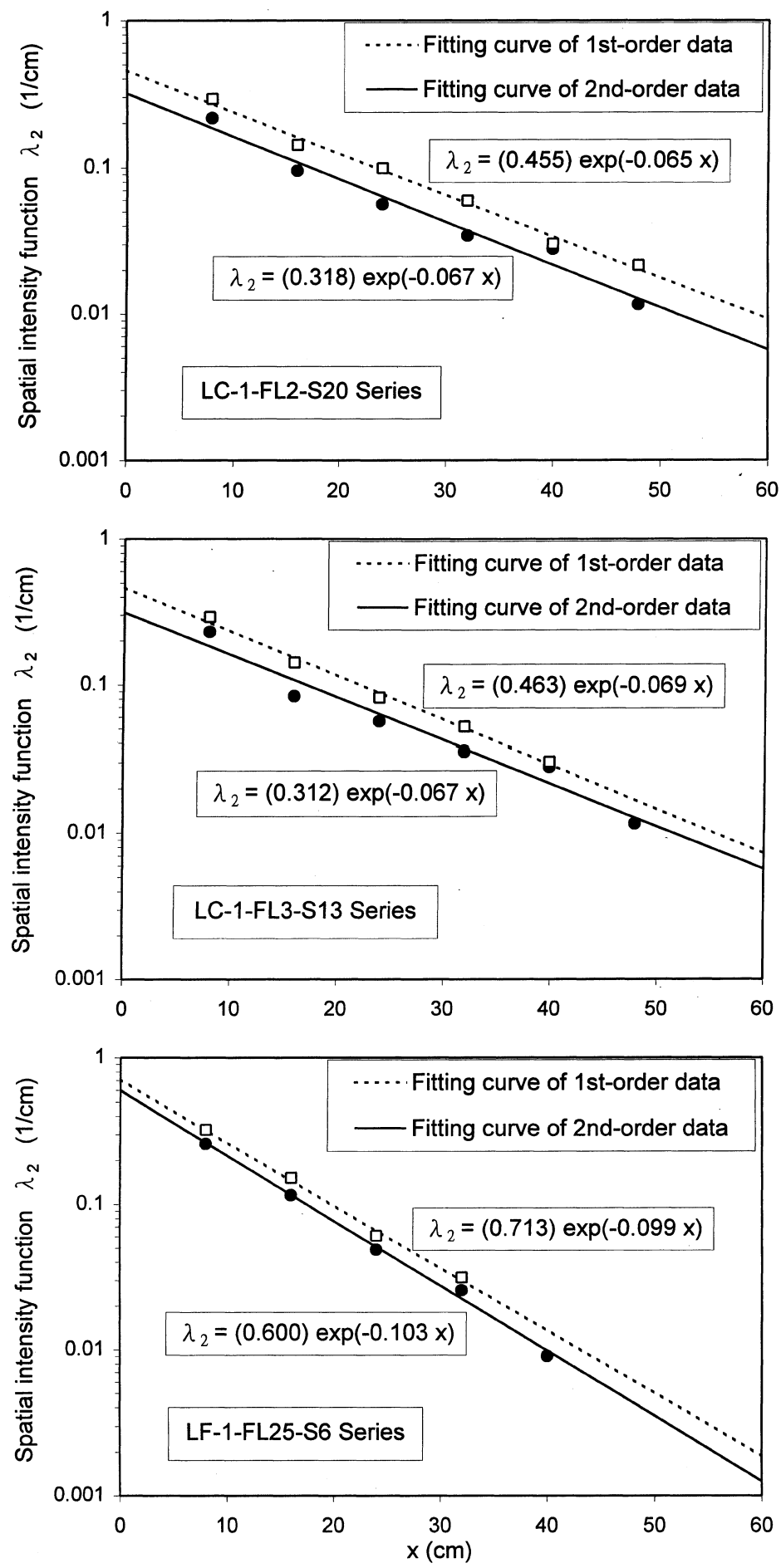

Fig. 2. Calculated data and fitting curves of spatial intensity function $\lambda_{2}$ ( $\square$ 1st-order data, - 2nd-order data) 
ment. The computed results and the experimental data are shown in Fig. 3. The second-order approximations apparently reveal better agreement with the experimental data. The Euclidean norm, a measure of the gross error between the computational and physical data, is defined as

$$
\|\mathrm{e}\|_{2}=\sqrt{\sum_{i=1}^{n} \mathrm{e}_{i}^{2}}
$$

in which $\mathrm{e}_{i}$ is the difference between the $i$ th data and the computational result, $n$ is the number of measured data (Conte and de Boor, 1980). The magnitudes of the Euclidean norm for all trials are summarized in Table 3. The gross errors of the second-order approximations are consistently smaller than the errors of the firstorder approximations. The reduction of the gross error for a single trial is of the order of $12 \%$ to $61 \%$, with an average of $47 \%$. Figure 3 also reveals that the magnitudes of the first-order CDF are larger than the magnitudes of the secondorder CDF. An inspection on Eq. (1) indicates that when $\Lambda_{2}$ is increasing, the growth of the compound infinite series is faster than the decay of the exponential term. As a whole, the product of the compound infinite series and the exponential terms in Eq. (1) is greater for larger $\Lambda_{2}$ (or $\left.\lambda_{2}\right)$.

\section{5}

\section{Error analysis}

The "error" is defined as the difference between the exact magnitude of a quantity (e.g. physical data) and its approximate value (e.g. simulated or computed result). The overall error of the estimated parameter or the cumulative probability distribution, in essence, contains two portions. The first portion originates from the underlying assumptions of the NHP model. The second portion arises from the approximation method. Discussion of the first error involves an examination of the rationality and applicability of the model (Hung and Shen, 1972). The main focus of this investigation is the computation error resulting from the approximate parameters. The experimental error is, however, not in the scope of the present study.

\section{1}

\section{Error of the spatial intensity function}

To evaluate the error of the spatial intensity function that is determined from a system of simplified equations, the error of such system should be considered at first. Theoretically, the error of the simplified CDF is the difference between the close and simplified forms of the equation. We do not have the exact form of $F_{t}(x+\Delta x) / F_{t}(x)$, nevertheless, the leading error term of the first-order simplified CDF can be estimated with its difference to the second-order simplified CDF (derivation shown in Appendix B), i.e.

$E_{C D F}^{(1)}=\left.\left[\frac{F_{t}(x+\Delta x)}{F_{t}(x)}\right]\right|^{(2)-(1)}=O\left(\Delta x^{2}\right)$

in which $E_{C D F}^{(1)}$ is the leading error term of the first-order simplified CDF, the superscript indicates the order of approximation. A quantity solved from a system with an error of $O\left(\Delta x^{2}\right)$ inherits an error of the same order (details shown in Appendix B), thus 

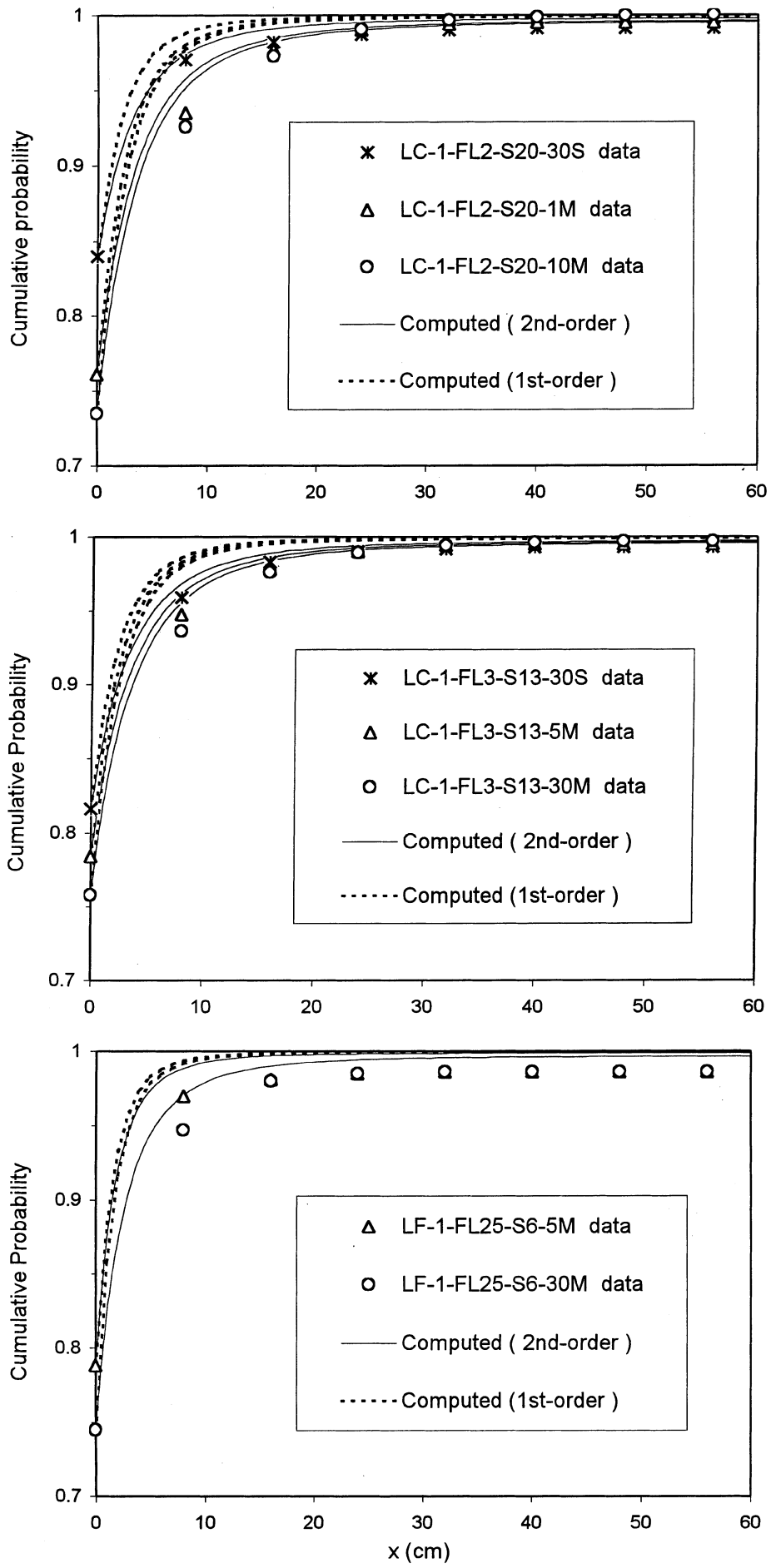

Fig. 3. Experimental data and computed cumulative probability distributions using the first- and second-order approximated parameters 
Table 3. Euclidean norm of computed results and percentage of reduced gross error for second-order results

\begin{tabular}{lllll}
\hline Experiment & \multicolumn{2}{l}{ Euclidean norm $\|\mathrm{e}\|_{2}$} & $\begin{array}{l}\text { Percentage of } \\
\text { reduced error } \\
{[(1)-(2)] /(1)}\end{array}$ & $\begin{array}{l}\text { Average } \\
\text { percentage of } \\
\text { reduced error }\end{array}$ \\
\cline { 2 - 4 } & $\begin{array}{l}\text { 1st-order } \\
(1)\end{array}$ & $\begin{array}{l}\text { 2nd-order } \\
(2)\end{array}$ & & \\
\hline LC-1-FL2-S20-30S & 0.032 & 0.018 & $43 \%$ & \\
LC-1-FL2-S20-1M & 0.050 & 0.024 & $53 \%$ & $49 \%$ \\
LC-1-FL2-S20-10M & 0.057 & 0.029 & $49 \%$ & \\
LC-1-FL3-S13-30S & 0.034 & 0.014 & $60 \%$ & $59 \%$ \\
LC-1-FL3-S13-5M & 0.042 & 0.016 & $61 \%$ & \\
LC-1-FL3-S13-30M & 0.049 & 0.021 & $58 \%$ & $27 \%$ \\
LF-1-FL25-S6-5M & 0.043 & 0.038 & $12 \%$ & $47 \%$ \\
LF-1-FL25-S6-30M & 0.057 & 0.034 & $41 \%$ & \\
& & & &
\end{tabular}

$a_{1}^{(1)}=\hat{a}_{1}+O\left(\Delta x^{2}\right)$

in which $\hat{a}_{1}$ is the close form solution of $a_{1}$. Using Eqs. (A-4) and (10), one can express the magnitude of the error associated with the first-order spatial intensity function as:

$\mathrm{e}^{(1)}=\hat{\lambda}_{2}-\lambda_{2}^{(1)}=O(\Delta x)$

in which $\hat{\lambda}_{2}$ is the exact magnitude of the spatial intensity function. The following relations also hold for the ith-order approximation method (Wu and Wang,

1997):

$E_{\mathrm{CDF}}^{(i)}=O\left(\Delta x^{i+1}\right)$

$\mathrm{e}^{(i)}=O\left(\Delta x^{i}\right)$

where $E_{\mathrm{CDF}}^{(i)}$ and $\mathrm{e}^{(\mathrm{i})}$ are the leading error of the $i$ th-order simplified CDF and the error of the spatial intensity function determined by the $i$ th-order approximation technique, respectively.

\section{2}

\section{Error of the cumulative probability distribution}

As shown in Appendix B, the error of the cumulative probability computed with the first-order spatial intensity function is of the first order of $\Delta x$, i.e.

$E^{(1)}=\hat{F}_{t}(x)-F_{t}^{(1)}(x)=O(\Delta x)$

in which $\hat{F}_{t}(x)$ and $F_{t}^{(1)}(x)$ are the exact magnitude and first-order approximation of the CDF at location $x$. The general form of the error for the ith-order approximation method can be expressed as the following (Wu and Wang, 1997):

$E^{(i)}=\hat{F}_{t}(x)-F_{t}^{(i)}(x)=O\left(\Delta x^{i}\right)$ 
The error analysis shows that the higher-order approximation method inherently leads to a higher-order error in terms of the finite increment. For a sufficiently small $\Delta x$, the magnitude of the error is significantly reduced as the higher-order method is used. Our results indicate that the second-order scheme eliminates nearly $50 \%$ of the CDF error of the first-order approximation.

\section{6}

\section{Conclusions}

This paper presents higher-order approximation techniques for estimating stochastic parameter of the non-homogeneous Poisson model used for modeling sediment transport. The spatial intensity function is estimated and thus the cumulative probability distribution of sediment displacement is calculated. The first- and second-order approximations are compared. The following conclusions can be drawn from this study:

(1) For the ith-order approximation method, the number of equations to be solved is $2 i$ and the number of locations where the physical data are to be gathered is $2 i+1$. The extra effort required for the higher-order scheme ensures improved accuracy of the estimated parameters, and therefore, the computed probability distributions of sediment displacement. The secondorder approximation technique leads to a $47 \%$ reduction of the CDF error of the first-order approximation. This improvement is of practical significance for a critical estimation of the sediment intrusion into spawning gravels.

(2) The spatial intensity function is of exponential distribution along the distance from the source. This function can be represented by Eq. (7), in which the exponent $b$ is nearly identical for the first- and second-order curves, yet the coefficient $a$ is greater for the first-order curve. The monotonously descending trend of the spatial intensity function (i.e. the increasing trend of the step length) is due to the increasing free void space for sediment particles to move through.

(3) For the ith-order approximation scheme, the leading error of the simplified CDF (as listed in Table 1) is of the order of $O\left(\Delta x^{i+1}\right)$; the spatial intensity function solved from a system of such simplified equations inherits an error of $O\left(\Delta x^{i}\right)$. The CDF error of the ith-order approximation is in a magnitude of $O\left(\Delta x^{i}\right)$.

\section{References}

Conte SD, de Boor C (1980) Elementary numerical analysis. New York: McGraw-Hill Cooper AC (1965) The effects of transported stream sediments on the survival of sockeye and pink salmon eggs and alevin. New Westminster, BC: International Pacific Salmon Fisheries Commission

Crickmore MJ, Lean GH (1962) The measurement of sand transport by the time-integration method with radioactive tracers. Proc. Roy. Soc. London A270, 27-47

Kreyszig E (1983) Advanced engineering mathematics. New York: John Wiley \& Sons Ltd Einstein HA (1937) Bed load transport as a probability problem. Dr. Sc. thesis, Federal Institute of Tech., Zurich, Switzerland

Hubbell DW, Sayre WW (1964) Sand transport studies with radioactive tracers. J. Hydraul. Div.-ASCE 90(HY3), 39-68

Hung CS, Shen HW (1972) Research in stochastic models for bed-load transport. In: Shen HW (ed.) River mechanics II, Appendix B. Fort Collins: Water Resources Publication Jobson HE, Carey WP (1989) Interaction of fine sediment with alluvial streambeds. Water Resour. Res. 25, 135-140

Joy DM, Lennox WC, Kouwen N (1993) Stochastic model of particulate transport in porous medium. J. Hydraul. Eng.-ASCE 119(7), 846-861 
Lisle TE (1989) Sediment transport and resulting deposition in spawning gravels, north coastal California. Water Resour. Res. 25, 1303-1319

Milhous RT (1982) Effect of sediment transport and flow regulation on the ecology of gravel-bed rivers. In: Hey RD, Bathurst JC, Thorne CR (eds.) Gravel-bed rivers. New York: John Wiley \& Sons Ltd

Shen HW, Cheong HF (1980) Stochastic sediment bed load models. In: Shen HW, Kikkawa $\mathrm{H}$ (eds.) Application of stochastic processes in sediment transport. Fort Collins: Water

Resources Publication

Shen HW, Todorovic P (1971) A general stochastic model for the transport of sediment bed material. In: Chiu C-L (ed.) Stochastic hydraulics. Pittsburgh: University of Pittsburgh Press Vukmirovic V, Wilson G Jr (1977) Bed load movement as random process. In: Hjorth P, Jönsson L Larson P (eds.) Hydraulic problems solved by stochastic methods. Fort Collins: Water Resources Publication

Wu F-C (1993) Stochastic modeling of sediment intrusion into gravel beds. Ph.D thesis, University of California, Berkeley, California

Wu F-C, Shen HW (1998) First-order estimation of stochastic parameters of a sediment transport model. Paper accepted for publication in J. Hydraul. Res.-IAHR

Wu F-C, Wang C-K (1997) A study on the second-order finite difference approximation of the stochastic model for sediment infiltration. NSC research report. Taipei: Dept. of Agricultural Engineering, National Taiwan University

Yang CT, Sayre WW (1971) Stochastic model for sand dispersion. J. Hydraul. Div.-ASCE 97(HY2), 265-288

\section{Appendix A}

\section{Derivation of Eqs. (2)-(5)}

To express $F_{t}(x+\Delta x)$, one requires expanding of $\left[\Lambda_{2}(x+\Delta x)\right]^{j}$ in which $\Lambda_{2}(x+\Delta x)$ is represented by Taylor series. The higher-order terms of $\Delta x$ (namely $\Delta x^{3}, \Delta x^{4}, \ldots$, etc.) in Taylor's expansions and the $j$ th-degree polynomial are negligible if the finite increment $\Delta x$ is sufficiently small, i.e.

$$
\begin{aligned}
{\left[\Lambda_{2}(x+\Delta x)\right]^{j}=} & {\left[\Lambda_{2}(x)+(\Delta x) \cdot \Lambda_{2}^{\prime}(x)+\left(\Delta x^{2}\right) \cdot \frac{\Lambda_{2}^{\prime \prime}(x)}{2 !}\right]^{j} } \\
= & {\left[\Lambda_{2}(x)\right]^{j}+j\left[\Lambda_{2}(x)\right]^{j-1}\left[(\Delta x) \cdot \Lambda_{2}^{\prime}(x)+\left(\Delta x^{2}\right) \cdot \frac{\Lambda_{2}^{\prime \prime}(x)}{2}\right] } \\
& +\frac{j(j-1)}{2}\left[\Lambda_{2}(x)\right]^{j-2}\left[(\Delta x) \cdot \Lambda_{2}^{\prime}(x)\right]^{2}
\end{aligned}
$$

Using Eqs. (1) and (A-1), one can express $F_{t}(x+\Delta x)$ as the following:

$$
\begin{aligned}
& F_{t}(x+\Delta x) \\
& =\exp \left[-\Lambda_{1}(t)\right] \cdot \exp \left[-\Lambda_{2}(x)\right] \cdot \exp \left[-(\Delta x) \cdot \Lambda_{2}^{\prime}(x)-\left(\Delta x^{2}\right) \cdot \frac{\Lambda_{2}^{\prime \prime}(x)}{2}\right] \\
& \quad \sum_{n=0}^{\infty} \sum_{j=n}^{\infty} \frac{\left[\Lambda_{1}(t)\right]^{n}}{n !}\left\{\frac{\left[\Lambda_{2}(x)\right]^{j}}{j !}+\frac{j\left[\Lambda_{2}(x)\right]^{j-1}\left[(\Delta x) \cdot \Lambda_{2}^{\prime}(x)+\left(\Delta x^{2}\right) \cdot \frac{\Lambda_{2}^{\prime \prime}(x)}{2}\right]}{j !}\right. \\
& \left.+\frac{\frac{j(j-1)}{2}\left[\Lambda_{2}(x)\right]^{j-2}\left[(\Delta x) \cdot \Lambda_{2}^{\prime}(x)\right]^{2}}{j !}\right\}
\end{aligned}
$$

We now temporarily focus our attention on the second item in the last braces of (A-2). Recognizing the fact that sediment transport is unidirectional and using 
the mathematical description of the compound NHP process (Shen and Todorovic, 1971), one can develop a concise form as follows:

$$
\begin{array}{r}
\exp \left[-\Lambda_{1}(t)\right] \cdot \exp \left[-\Lambda_{2}(x)\right] \cdot \exp \left[-(\Delta x) \cdot \Lambda_{2}^{\prime}(x)-\left(\Delta x^{2}\right) \cdot \frac{\Lambda_{2}^{\prime \prime}(x)}{2}\right] \\
\cdot \sum_{n=0}^{\infty} \sum_{j=n}^{\infty} \frac{\left[\Lambda_{1}(t)\right]^{n}}{n !} \frac{j\left[\Lambda_{2}(x)\right]^{j-1}\left[(\Delta x) \cdot \Lambda_{2}^{\prime}(x)+\left(\Delta x^{2}\right) \cdot \frac{\Lambda_{2}^{\prime \prime}(x)}{2}\right]}{j !} \\
=\exp \left(-a_{1}-a_{2}\right) \cdot\left(a_{1}+a_{2}\right) \cdot \exp \left[-\Lambda_{1}(t)\right] \cdot \exp \left[-\Lambda_{2}(x)\right] \\
\cdot \quad\left\{\frac{\left[\Lambda_{1}(t)\right]^{0}}{0 !} \cdot \sum_{p=-1}^{\infty} \frac{\left[\Lambda_{2}(x)\right]^{p}}{p !}+\sum_{n=1}^{\infty} \sum_{q=n-1}^{\infty} \frac{\left[\Lambda_{1}(t)\right]^{n}}{n !} \frac{\left[\Lambda_{2}(x)\right]^{q}}{q !}\right\} \\
=\exp \left(-a_{1}-a_{2}\right) \cdot\left(a_{1}+a_{2}\right) \cdot\left[P\left(E_{0}^{t_{0}, t}\right)+\sum_{n=1}^{\infty} P\left(X_{n-1} \leq x\right) P\left(E_{n}^{t_{0}, t}\right)\right]
\end{array}
$$

in which $a_{1}$ and $a_{2}$ are given in Table $1 ; X_{n-1}$ is the distance traveled after making $n-1$ steps; $E_{0}^{t_{0}, t}$ and $E_{n}^{t_{0}, t}$ are the events of making respectively 0 and $n$ steps within the period $\left[t_{0}, t\right]$.

Next, one can similarly simplify the expanded form associated with the third item in the last braces of (A-2) as follows:

$$
\begin{aligned}
& \exp \left[-\Lambda_{1}(t)\right] \cdot \exp \left[-\Lambda_{2}(x)\right] \cdot \exp \left[-(\Delta x) \cdot \Lambda_{2}^{\prime}(x)-\left(\Delta x^{2}\right) \cdot \frac{\Lambda_{2}^{\prime \prime}(x)}{2}\right] \\
& \cdot \sum_{n=0}^{\infty} \sum_{j=n}^{\infty} \frac{\left[\Lambda_{1}(t)\right]^{n}}{n !}\left\{\frac{\frac{j(j-1)}{2}\left[\Lambda_{2}(x)\right]^{j-2}\left[(\Delta x) \cdot \Lambda_{2}^{\prime}(x)\right]^{2}}{j !}\right\} \\
& =\exp \left(-a_{1}-a_{2}\right) \cdot \frac{a_{1}^{2}}{2} \cdot \exp \left[-\Lambda_{1}(t)\right] \cdot \exp \left[-\Lambda_{2}(x)\right] \\
& \left.\quad \cdot \frac{\left[\Lambda_{1}(t)\right]^{0}}{0 !} \sum_{p=-2}^{\infty} \frac{\left[\Lambda_{2}(x)\right]^{p}}{p !}+\frac{\left[\Lambda_{1}(t)\right]^{1}}{1 !} \sum_{q=-1}^{\infty} \frac{\left[\Lambda_{2}(x)\right]^{q}}{q !}+\sum_{n=2}^{\infty} \sum_{r=n-2}^{\infty} \frac{\left[\Lambda_{1}(t)\right]^{n}}{n !} \frac{\left[\Lambda_{2}(x)\right]^{r}}{r !}\right\} \\
& =\exp \left(-a_{1}-a_{2}\right) \cdot \frac{a_{1}^{2}}{2} \cdot\left[P\left(E_{0}^{t_{0}, t}\right)+P\left(E_{1}^{t_{0}, t}\right)+\sum_{n=2}^{\infty} P\left(X_{n-2} \leq x\right) P\left(E_{n}^{t_{0}, t}\right)\right] \quad(\mathrm{A}-4)
\end{aligned}
$$

where the definitions of the corresponding terms are similar to those provided previously. Substituting Eqs. (1), (A-3) and (A-4) into Eq. (A-2) yields

$$
\begin{aligned}
F_{t}(x+\Delta x)= & \exp \left(-a_{1}-a_{2}\right) \\
& \cdot\left\{F_{t}(x)+\left(a_{1}+a_{2}\right) \cdot\left[P\left(E_{0}^{t_{0}, t}\right)+\sum_{n=1}^{\infty} P\left(X_{n-1} \leq x\right) P\left(E_{n}^{t_{0}, t}\right)\right]\right. \\
& \left.+\frac{a_{1}^{2}}{2} \cdot\left[P\left(E_{0}^{t_{0}, t}\right)+P\left(E_{1}^{t_{0}, t}\right)+\sum_{n=2}^{\infty} P\left(X_{n-2} \leq x\right) P\left(E_{n}^{t_{0}, t}\right)\right]\right\}
\end{aligned}
$$

Dividing (A-5) by Eq. (1) leads to Eq. (2). Following the same procedures by replacing $\Delta x$ with $-\Delta x, 2 \Delta x$, and $-2 \Delta x$, one can obtain Eqs. (3), (4), and (5), respectively. 


\section{Appendix B}

1

\section{Derivation of Eq. (9)}

Based on the simplified CDF given in Table 1, one can express the difference between the second- and first-order simplified CDF as:

$$
\begin{aligned}
E_{C D F}^{(1)}= & \left\{\exp \left(-a_{1}-a_{2}\right) \cdot\left[1+\left(a_{1}+a_{2}\right) \cdot b_{1}+\frac{1}{2} a_{1}^{2} \cdot b_{2}\right]\right\} \\
& -\left[\exp \left(-a_{1}\right) \cdot\left(1+a_{1} \cdot b_{1}\right)\right]
\end{aligned}
$$

One can further substitute the exponential function with Maclaurin series (Kreyszig, 1983) and rearrange Eq. (B-1) as a function of $\Delta x$, i.e.

$$
\begin{aligned}
E_{C D F}^{(1)}= & \left\{\left[1-a_{1}+\frac{a_{1}^{2}}{2}+O\left(a_{1}^{3}\right)\right] \cdot\left[1-a_{2}+\frac{a_{2}^{2}}{2}+O\left(a_{2}^{3}\right)\right]\right. \\
& \left.\cdot\left(1+a_{1} \cdot b_{1}+a_{2} \cdot b_{1}+\frac{1}{2} a_{1}^{2} \cdot b_{2}\right)\right\} \\
& -\left\{\left[1-a_{1}+\frac{a_{1}^{2}}{2}+O\left(a_{1}^{3}\right)\right] \cdot\left(1+a_{1} \cdot b_{1}\right)\right\} \\
= & \phi \cdot \Delta x^{2}+O\left(\Delta x^{3}\right)
\end{aligned}
$$

in which

$\phi=\frac{b_{2}}{2}\left(\Lambda_{2}^{\prime}\right)^{2}+\frac{b_{1}-1}{2}\left(\Lambda_{2}^{\prime \prime}\right)$

\section{2}

\section{Derivation of Eq. (10)}

For the first-order approximation method, $a_{1}$ and $b_{1}$ are solved from a system of the following form (Wu and Shen, 1998):

$$
\begin{aligned}
& {\left[\frac{F_{t}(x+\Delta x)}{F_{t}(x)}\right]^{(1)}=f_{1}\left(a_{1}, b_{1}\right)} \\
& {\left[\frac{F_{t}(x-\Delta x)}{F_{t}(x)}\right]^{(1)}=f_{2}\left(a_{1}, b_{1}\right)}
\end{aligned}
$$

in which the explicit form of $f_{1}\left(a_{1}, b_{1}\right)$ is shown in Table 1. From Eq. (B-2), one also knows that the following relationships exist for sufficiently small $\Delta x$ :

$$
\begin{aligned}
& {\left[\frac{F_{t}(x+\Delta x)}{F_{t}(x)}\right]^{(1)}=\left[\frac{F_{t}(x+\Delta x)}{F_{t}(x)}\right]^{(\infty)}-\phi \cdot \Delta x^{2}} \\
& {\left[\frac{F_{t}(x-\Delta x)}{F_{t}(x)}\right]^{(1)}=\left[\frac{F_{t}(x-\Delta x)}{F_{t}(x)}\right]^{(\infty)}-\phi \cdot \Delta x^{2}}
\end{aligned}
$$


Given $\hat{a}_{1}$ and $\hat{b}_{1}$ are the first two components of the close form solution to the system of the infinite-order simplified CDF equations. Such system is theoretically possible as the number $\alpha$, in Table 1, approaches to infinity. By virtue of Eq. (B-3), one can substitute the close form solution into Eq. (B-4) such that

$$
\begin{aligned}
& f_{1}\left(\hat{a}_{1}, \hat{b}_{1}\right)=\hat{f_{1}}=C_{1}-\hat{\phi} \cdot \Delta x^{2} \\
& f_{2}\left(\hat{a}_{1}, \hat{b}_{1}\right)=\hat{f_{2}}=C_{2}-\hat{\phi} \cdot \Delta x^{2}
\end{aligned}
$$

374 in which $C_{1}$ and $C_{2}$ are the exact magnitudes of $F_{t}(x+\Delta x) / F_{t}(x)$ and $F_{t}(x-\Delta x) / F_{t}(x)$, respectively; Eq. (B-2) gives $\hat{\phi}=\left[\hat{b}_{2}\left(\Lambda_{2}^{\prime}\right)^{2}+\left(\hat{b}_{1}-1\right)\left(\Lambda_{2}^{\prime \prime}\right)\right] / 2$.

However, the problem we are practically dealing with is to seek solutions from the following system of equations since $C_{1}$ and $C_{2}$ are determined from the physical data.

$$
\begin{aligned}
& f_{1}\left(a_{1}^{(1)}, b_{1}^{(1)}\right)=C_{1} \\
& f_{2}\left(a_{1}^{(1)}, b_{1}^{(1)}\right)=C_{2}
\end{aligned}
$$

or

$f_{1}\left(\hat{a}_{1}+e_{a}^{(1)}, \hat{b}_{1}+e_{b}^{(1)}\right)=C_{1}$

$f_{2}\left(\hat{a}_{1}+e_{a}^{(1)}, \hat{b}_{1}+e_{b}^{(1)}\right)=C_{2}$

where $e_{a}^{(1)}$ and $e_{b}^{(1)}$ are the errors between $a_{1}^{(1)}$ and $\hat{a}_{1}, b_{1}^{(1)}$ and $\hat{b}_{1}$, respectively. With Eq. (B-5) and Taylor series, one can reform Eq. (B-6) into the following if the first-order terms of $e_{a}^{(1)}$ and $e_{b}^{(1)}$ are solely considered:

$$
\begin{aligned}
& \hat{f_{1}}+\frac{\partial \hat{f}_{1}}{\partial \hat{a}_{1}} \cdot e_{a}^{(1)}+\frac{\partial \hat{f}_{1}}{\partial \hat{b}_{1}} \cdot e_{b}^{(1)}=\hat{f_{1}}+\hat{\phi} \cdot \Delta x^{2} \\
& \hat{f}_{2}+\frac{\partial \hat{f}_{2}}{\partial \hat{a}_{1}} \cdot e_{a}^{(1)}+\frac{\partial \hat{f}_{2}}{\partial \hat{b}_{1}} \cdot e_{b}^{(1)}=\hat{f_{2}}+\hat{\phi} \cdot \Delta x^{2}
\end{aligned}
$$

Solving (B-7) for $e_{a}^{(1)}$ and $e_{b}^{(1)}$ leads to

$$
\begin{aligned}
& e_{a}^{(1)}=\hat{\eta}_{1} \cdot \Delta x^{2} \\
& e_{b}^{(1)}=\hat{\eta}_{2} \cdot \Delta x^{2}
\end{aligned}
$$

in which

$$
\begin{aligned}
& \hat{\eta}_{1}=\frac{\left[-\left(\partial \hat{f}_{1} / \partial \hat{b}_{1}\right)+\left(\partial \hat{f}_{2} / \partial \hat{b}_{1}\right)\right] \cdot \hat{\phi}}{\left(\partial \hat{f}_{1} / \partial \hat{a}_{1}\right)\left(\partial \hat{f}_{2} / \partial \hat{b}_{1}\right)-\left(\partial \hat{f_{1}} / \partial \hat{b}_{1}\right)\left(\partial \hat{f}_{2} / \partial \hat{a}_{1}\right)} \\
& \hat{\eta}_{2}=\frac{\left[\left(\partial \hat{f}_{1} / \partial \hat{a}_{1}\right)-\left(\partial \hat{f}_{2} / \partial \hat{a}_{1}\right)\right] \cdot \hat{\phi}}{\left(\partial \hat{f}_{1} / \partial \hat{a}_{1}\right)\left(\partial \hat{f}_{2} / \partial \hat{b}_{1}\right)-\left(\partial \hat{f}_{1} / \partial \hat{b}_{1}\right)\left(\partial \hat{f}_{2} / \partial \hat{a}_{1}\right)}
\end{aligned}
$$


Derivation of Eq. (13)

By virtue of Eq. (B-8) and the definition of $a_{1}$, one can write an equation relating $\lambda_{2}^{(1)}$ and $\hat{\lambda}_{2}$ :

$\lambda_{2}^{(1)}=\hat{\lambda}_{2}+\hat{\eta}_{1} \cdot \Delta x$

$\lambda_{2}^{(1)}$ is substituted into Eq. (1) to evaluate the cumulative probability distribution of sediment displacement, i.e.

$$
\begin{aligned}
F_{t}^{(1)}(x)= & \exp \left[-\Lambda_{1}\right] \cdot \exp \left[-\hat{\Lambda}_{2}(x)-\Delta x \cdot \hat{\mathrm{I}}_{1}(x)\right] \\
& \cdot \sum_{n=0}^{\infty} \sum_{j=n}^{\infty} \frac{\left[\Lambda_{1}\right]^{n}}{n !} \frac{\left[\hat{\Lambda}_{2}(x)+\Delta x \cdot \hat{\mathrm{I}}_{1}(x)\right]^{j}}{j !}
\end{aligned}
$$

in which

$$
\hat{\Lambda}_{2}(x)+\Delta x \cdot \hat{\mathrm{I}}_{1}(x)=\int_{x_{0}}^{x}\left[\hat{\lambda}_{2}(\xi)+\Delta x \cdot \hat{\eta}_{1}(\xi)\right] \mathrm{d} \xi
$$

Substituting the exponential function in Eq. (B-10) with Maclaurin series and expanding the last $j$ th-power term leads to

$$
\begin{aligned}
F_{t}^{(1)}(x)= & {\left[1-\Delta x \cdot \hat{\mathrm{I}}_{1}(x)+O\left(\Delta x^{2}\right)\right] \cdot\left\{\exp \left[-\Lambda_{1}\right] \cdot \exp \left[-\hat{\Lambda}_{2}(x)\right]\right.} \\
& \cdot \sum_{n=0}^{\infty} \sum_{j=n}^{\infty} \frac{\left[\Lambda_{1}\right]^{n}}{n !} \frac{\left[\hat{\Lambda}_{2}(x)\right]^{j}}{j !}+\Delta x \cdot \hat{\mathrm{I}}_{1}(x) \cdot \exp \left[-\Lambda_{1}\right] \cdot \exp \left[-\hat{\Lambda}_{2}(x)\right] \\
& \left.\cdot \sum_{n=0}^{\infty} \sum_{j=n}^{\infty} \frac{\left[\Lambda_{1}\right]^{n}}{n !} \frac{j\left[\hat{\Lambda}_{2}(x)\right]^{j-1}}{j !}+O\left(\Delta x^{2}\right)\right\}
\end{aligned}
$$

One can further simplify Eq. (B-11) as the following:

$F_{t}^{(1)}(x)=\hat{F}_{t}(x)+\psi \cdot \Delta x+O\left(\Delta x^{2}\right)$

in which

$$
\begin{aligned}
& \psi=\hat{\mathrm{I}}_{1}(x) \cdot\left[\hat{\mathrm{K}}(x)-\hat{F}_{t}(x)\right] \\
& \hat{F}_{t}(x)=\exp \left[-\Lambda_{1}\right] \cdot \exp \left[-\hat{\Lambda}_{2}(x)\right] \cdot \sum_{n=0}^{\infty} \sum_{j=n}^{\infty} \frac{\left[\Lambda_{1}\right]^{n}}{n !} \frac{\left[\hat{\Lambda}_{2}(x)\right]^{j}}{j !} \\
& \hat{\mathrm{K}}(x)=\exp \left[-\Lambda_{1}\right] \cdot \exp \left[-\hat{\Lambda}_{2}(x)\right] \cdot \sum_{n=0}^{\infty} \sum_{j=n}^{\infty} \frac{\left[\Lambda_{1}\right]^{n}}{n !} \frac{\left[\hat{\Lambda}_{2}(x)\right]^{j-1}}{j !}
\end{aligned}
$$

